\title{
The envelope of human endogenous retrovirus in neuro-inflammation
}

\author{
Hei-Lanne Dougier*1, Christina Lomparski ${ }^{1}$, Christian Villiers ${ }^{1}$, \\ Alain Duperray ${ }^{1}$, Corinne Bernard ${ }^{2}$, Evelyne Jouvin-Marche ${ }^{1}$, Hervé Perron ${ }^{2}$ \\ and Patrice N Marche ${ }^{1}$
}

Address: ${ }^{1}$ Université J Fourier, Inserm U823, Institut Albert Bonniot, Grenoble, 3800, France and ${ }^{2}$ Geneuro SA, Plan les Ouates, 1228, Switzerland

* Corresponding author

from Frontiers of Retrovirology: Complex retroviruses, retroelements and their hosts

Montpellier, France. 2I-23 September 2009

Published: 24 September 2009

Retrovirology 2009, 6(Suppl 2):P53 doi:10.1186/I742-4690-6-S2-P53

This abstract is available from: http://www.retrovirology.com/content/6/S2/P53

(c) 2009 Dougier et al; licensee BioMed Central Ltd.

Several viruses are known to interact with the host defences either to escape from the immune responses or to gain advantage of inflammatory mediators to survive. Human endogenous retroviruses (HERV) are integrated and are estimated represent up to $8 \%$ o the human genome. An exogenous virus from HERV. W family was initially isolated from brain cells of patients suffering of multiple sclerosis and named MSRV [1]. Several lines of evidence support that its envelope protein (ENV) or its soluble extra-cellular subunit (ENV-SU) contributes to inflammation associated with the disease: 1) ENV promotes polyclonal expansion of $\mathrm{T}$ lymphocytes [2], 2) ENV-SU induces human monocytes and dendritic cells (DC) to produce inflammatory cytokines through engagement of CD14 and TLR4 [3].

In order to study in vivo effects of MSRV, mice were treated for experimental allergic encephalitis (EAE) induction, a mouse model for MS, after antigenic myelin peptide immunisation either with complete Freund's adjuvant or ENV-SU. Clinical score showed significant EAE symptoms in both mice but no symptoms in control mice receiving no adjuvant or no ENV (Fig. 1). Cultures of splenocytes from either ENV-SU or adjuvant treated mice, recalled with the myelin antigen, led to IFN- $\gamma$ production (not shown), indicating $\mathrm{T}$ lymphocyte reactivity towards the myelin antigen.

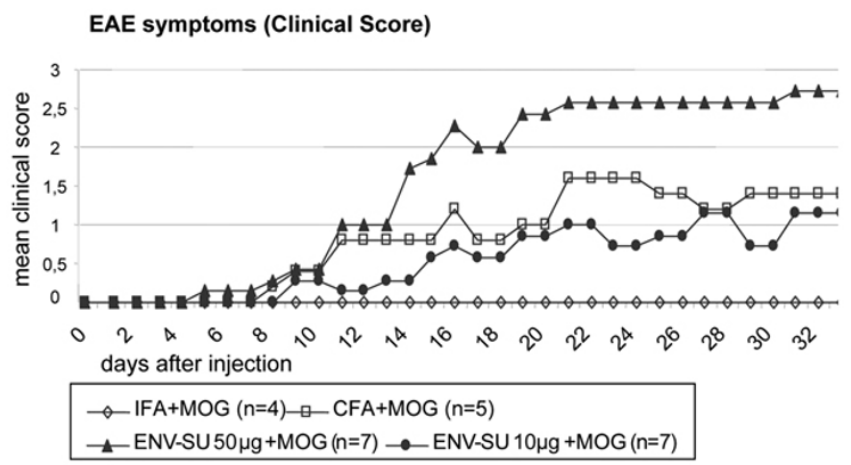

Figure I

Clinical score follow-up of C57BL/6 mice injected with MOG 35-55 and IFA, CFA or ENV. Mean values were calculated for each group of mice.

To characterise the mode of action of ENV in mice, DC were cultured from C57BL/ 6 bone marrow and incubated with ENV-SU. DC secreted amounts of IL-6 (Fig. 2) and IL12 p70 (not shown) dose-dependant of the ENV-SU. Flow cytometry analysis for CMH-II, CD11c, B7.1 and B7.2 molecules DC demonstrated that DC were activated and underwent further differentiation upon ENV-SU exposure comparable to other known stimulations. DC derived from TLR4 (not shown) or CD14 deficient mice (Fig. 3) were unable to respond to ENV-SU stimulation, arguing that, as found in humans, TLR4/CD14 pathway is involved in DC responses in mice. 


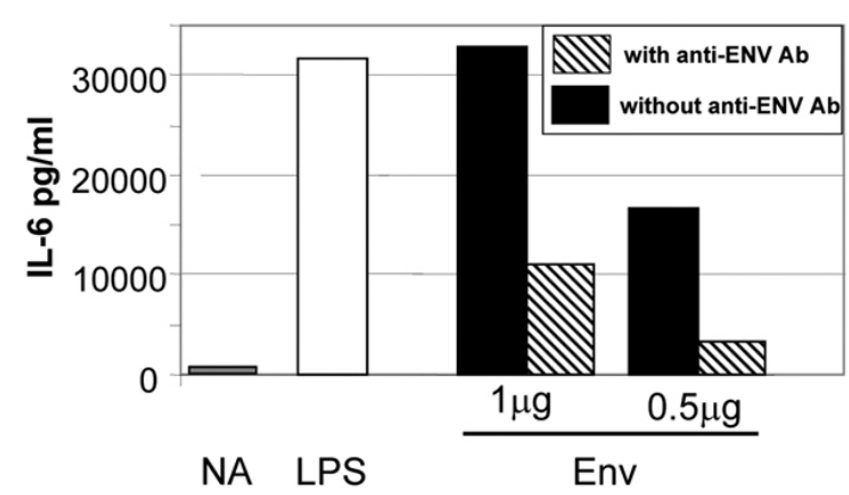

Figure 2

Activation of mouse dendritic cells by the envelope. Bone marrow derived DC were incubated with either $0.5 \mu \mathrm{g} /$ $\mathrm{ml}$ or $1 \mu \mathrm{g} / \mathrm{ml}$ of ENV-SU for $24 \mathrm{~h}$ and IL- 6 was measured by ELISA. Before incubation with the cells, ENV-SU is incubated, or not, with the anti-ENV antibodies during $I \mathrm{~h}$ at $4^{\circ} \mathrm{C}$. Control samples are incubated with ENV-SU alone, with LPS or without stimulation (NA).

\section{Conclusion}

by promoting inflammatory response through CD14/ TLR4 pathway, the envelope of MSRV/HERV contributes to EAE in mice and thus may be one of the key actors of MS etiology in humans.

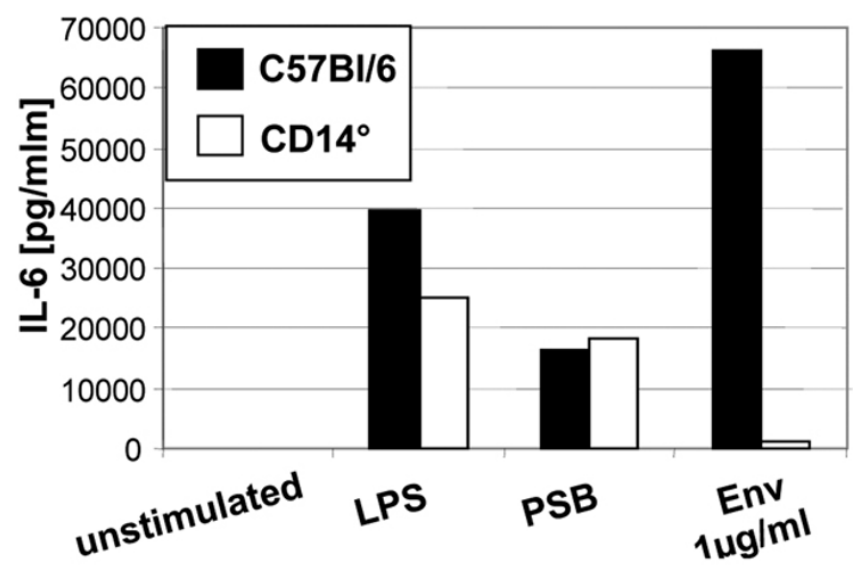

Figure 3

Activation of mouse dendritic cells by the envelope depends on CDI4. (see legend Fig2). $\mathrm{CDI} 4^{\circ}=\mathrm{CDI} 4$ deficient mouse.

\section{References}

I. Perron H, Garson JA, Bedin F, Beseme F, Paranhos-Baccala G, Komurian-Pradel F, Mallet F, Tuke PW, Voisset C, Blond JL, et al:: Molecular identification of a novel retrovirus repeatedly isolated from patients with multiple sclerosis. The Collaborative Research Group on Multiple Sclerosis. Proc Natl Acad Sci USA 1997, 94:7583-7588.

2. Perron H, Jouvin-Marche E, Michel M, Ounanian-Paraz A, Camelo S, Dumon A, Jolivet-Reynaud C, Marcel F, Souillet Y, Borel E, et al.: Multiple sclerosis retrovirus particles and recombinant envelope trigger an abnormal immune response in vitro, by inducing polyclonal Vbetal6 T-lymphocyte activation. Virology 2001 , 287:321-332.

3. Rolland A, Jouvin-Marche E, Viret C, Faure M, Perron H, Marche PN: The envelope protein of a human endogenous retrovirus- $W$ family activates innate immunity through CDI4/TLR4 and promotes ThI-like responses. J Immunol 2006, I 76:7636-7644. 